\title{
Modeling Ion Beam Induced Secondary Electrons
}

$\underline{\text { U. Huh }}^{1}$, V. Iberi ${ }^{1}$, W. Cho ${ }^{2}$, R. Ramachandra ${ }^{3}$, and D. C. Joy ${ }^{1,4}$

${ }^{1}$ Biochemistry and Cellular and Molecular Biology, University of Tennessee, Knoxville, TN 37996

${ }^{2}$ Electrical and Computer Engineering, University of Tennessee, Knoxville, TN 37996

${ }^{3}$ National Center for Microscopy and Imaging Research, University of California San Diego, La Jolla, CA 92093

${ }^{4}$ Center for Nanophase Materials Science, Oak Ridge National Laboratory, Oak Ridge, TN 37831

In the Helium Ion Microscope the incident ion beam interacts with the specimen to generate secondary electrons which then carry the desired image information to the detector for viewing. An essential requirement is to be able to determine the ion generated secondary electron (iSE) yield from the sample as a function of the incident ion energy and the choice of the material. Presently experimental iSE yield data for helium ions is limited to just 40 elements or so and a few binary compounds [1]. There is therefore a need for a detailed predictive model which can provide iSE yield data for a wide range of beam energies and for both pure elements and for compounds, which so far has not been possible.

In the published first generation version of this program the ion stopping power as a function of ion atomic weight was taken from published data and numerically stored for use. Instead of using multiple individual data tables of the stopping power we will exploit the "ASTAR" program, recently developed by Berger et al [2] at NIST, which computes ion stopping power data for arbitrary energies and target material compositions. This code, freely available at the NIST web site and can be run on any standard computer, is able to compute the stopping power profiles for any ion impinging on an arbitrary target material, across five orders of magnitude of the beam energy as shown in Figure 1 for $\mathrm{He}^{+}$ions.

As a starting point we have chosen to use the averaged value of the ASTAR plot for some 80 elements in the periodic table, and a number of binary compounds in the energy range 1 to $100 \mathrm{keV}$ from Figure 1. The ion energy range of interest presently extends from about $10 \mathrm{keV}$ to $100 \mathrm{keV}$ if ASTAR is used to compute stopping power data for all elements and other common materials. Then, if these plots are super-imposed one on other, it immediately gives a hint that there is very little difference in generic shape of the stopping power curve and absolute intensity within the energy range from $10 \mathrm{keV}$ to 100 $\mathrm{keV}$ and the overlaid plots indicates all the predicted data falls closely around the same for images up to energies in excess of $1 \mathrm{MeV}$.

Figure 2 shows stopping power data for carbon, silicon, carbon dioxide, and silicon dioxide are in good agreement to the previously discussed aspects. $1^{\text {st }}$ approximation of the overlaid stopping power curves for $\mathrm{He}^{+}$ions within the range of interest, effective range of the beam in use, produces a "universal" stopping power curve for helium ion interactions with any given element yet compounds and at any energies as shown in Figure 3. With this stopping power data it is then possible for the IONiSE program to reliably predict parameters such as the beam range, and the secondary electron yield from not only pure elements but also compound materials, which so far has not been possible. This approximation approach fits the presently available published data very well. iSE data for compounds is very scarce but the few published examples are also in close agreement to this ASTAR approach. This result is not being proposed as a perfect solution to the problem of simulating ion beam induced images but, given the almost total absence of high quality experimental data, this approach is a big step forward. 

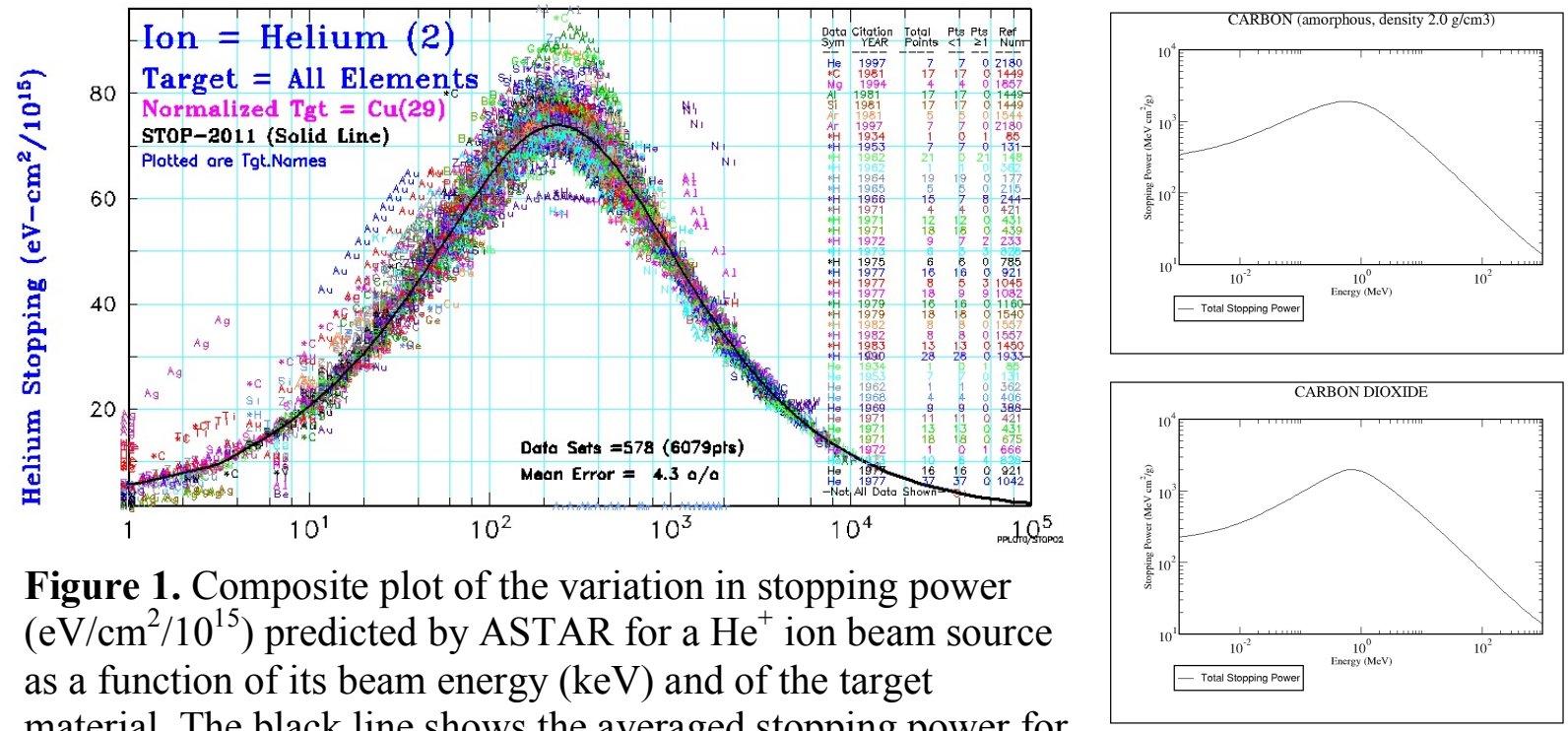

Figure 1. Composite plot of the variation in stopping power $\left(\mathrm{eV} / \mathrm{cm}^{2} / 10^{15}\right)$ predicted by ASTAR for a $\mathrm{He}^{+}$ion beam source as a function of its beam energy (keV) and of the target material. The black line shows the averaged stopping power for all the materials tested and as a function of ion energy $[2,3]$
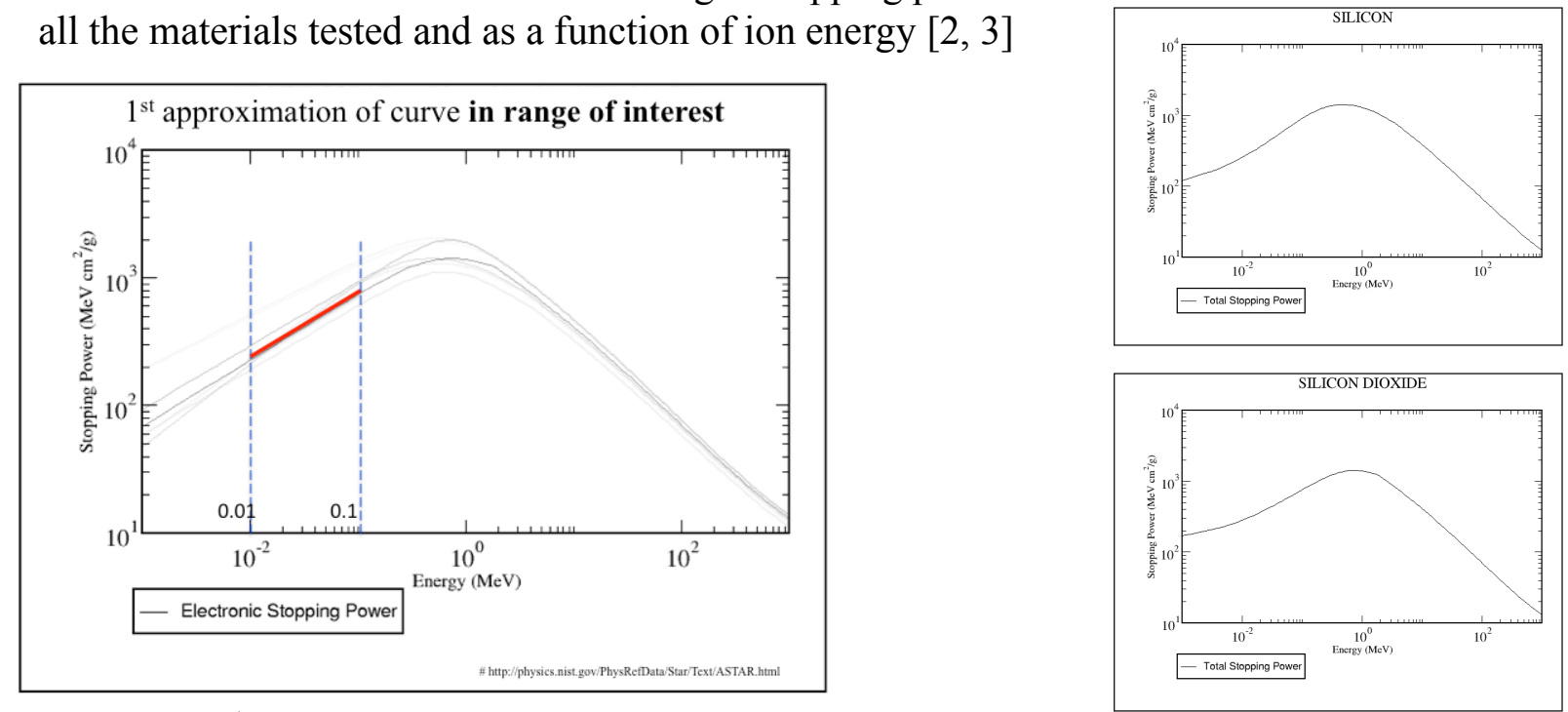

Figure 3. $1^{\text {st }}$ approximation of overlays of stopping power curves for $\mathrm{He}^{+}$ions in range of interest

Figure 2. Stopping power of $\mathrm{C}, \mathrm{CO}_{2}$, $\mathrm{Si}$ and $\mathrm{SiO}_{2}$ for $\mathrm{He}^{+}$ions [2]

\section{References}

[1] R. Ramachandra, B. Griffin, and D. Joy, "A model of secondary electron imaging in the helium ion scanning microscope," Ultramicroscopy, vol. 109, May 2009.

[2] J. S. C. M.J. Berger, M.A. Zucker, J. Chang. (2011). Stopping-Power and Range Tables for Electrons, Protons, and Helium Ions. Available: http://www.nist.gov/pml/data/star/index.cfm

[3] J. F. Ziegler. (2013). PARTICLE INTERACTIONS WITH MATTER. Available: http://www.srim.org/

[4] This works was partially supported by the Biochemistry and Cellular and Molecular Biology at The University of Tennessee. 